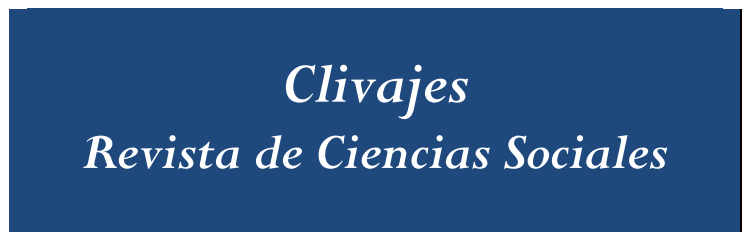

Clivajes. Revista de Ciencias Sociales ISSN: 2395-9495

https: / / goo.gl/Lpsy44

IIH-S, UV, México

Cecilia Sarabia Ríos y Nemesio Castillo Viveros

DeSARROLlO E INCIDENCIA DE LAS ORGANIZACIONES DE LA SOCIEDAD CIVIL EN CHIHUAHUA, MÉXICO

Clivajes. Revista de Ciencias Sociales. Año V, número 9, enero-junio 2018, pp. 50-70.

Instituto de Investigaciones Histórico-Sociales

Universidad Veracruzana. México

Recibido: 10-01-2017

Aceptado: 15-02-2017

Dictaminado: 17-11-2017

Publicado: 01-01-2018 


\title{
DESARRollo E INCIDENCIA DE LAS ORGANIZACIONES DE LA SOCIEDAD CiVIL EN CHIHUAHUA, MÉXICO
}

\author{
Cecilia Sarabia Ríos* \\ Nemesio Castillo Viveros ${ }^{* *}$
}

Resumen

Las Organizaciones de la Sociedad Civil en Chihuahua han desarrollado un proceso de reflexión e innovación en cuanto a estrategias de participación en el espacio público y en el posicionamiento de sus demandas sociales en la agenda oficial. En esta investigación, se analiza el origen y los cambios provocados por la implementación de las políticas económicas neoliberales en México y su correspondencia con los registrados en la relación entre el Estado y la sociedad civil, en particular, con sus organizaciones. Se expone el caso del Plan Estratégico de Juárez como ejemplo emblemático en los procesos de adaptación, innovación e incidencia en la toma de decisiones, y se argumenta a favor de una lógica de adaptación y resistencia ante las políticas neoliberales del Estado Mexicano, a través de la definición, negociación y renegociación con sus instituciones.

Palabras clave: Neoliberalismo y Sociedad civil, y Organizaciones

\section{INTRODUCCIÓN}

En México, la aplicación de políticas neoliberales, desde principios de la década de 1980, provocó un aumento en el número de organizaciones con presencia en la agenda pública en relación con los temas más importantes para el ámbito comunitario. Desde sus inicios, las Organizaciones de la Sociedad Civil (OSC) orientaron sus esfuerzos a la atención de una amplia gama de problemas sociales no atendidos por el Estado, como la drogadicción, las infecciones de transmisión sexual, las enfermedades terminales, la educación formal y no formal, la marginación y la pobreza, entre otros, es decir, las OSC asumieron a la responsabilidad de plantear y atender, por lo menos parcialmente, algunas de las problemáticas que requerían solución más urgente y no contaban (ni cuentan) con atención alguna por parte de las instituciones del Estado. En ese sentido, el rol de las OSC ha consistido, fundamentalmente, en proponer alternativas creativas para visibilizar y dar cobertura a dichas problemáticas; en algunos casos, incluso se han hecho cargo de trabajar en ellas directamente.

A menudo, las OSC son resultado de la organización política que busca mejorar las condiciones de calidad de vida en sus comunidades; sin embargo, como hemos mencionado, la

\footnotetext{
* El Colegio de la Frontera Norte A.C., México.

** Universidad Autónoma de Ciudad Juárez, México.
} 
instrumentación del modelo neoliberal, hace casi cuatro décadas, ha condicionado la manera en que operan los países en términos políticos, económicos y sociales. A raíz de ello, se han producido grandes transformaciones en la acción colectiva, en el campo social, que han impulsado a su vez cambios en los paradigmas que cobijan la labor de los actores sociales.

La influencia de las OSC en la esfera pública es multifactorial. En ocasiones tienen capacidad para posicionar los temas que enarbolan; en otras, despliegan estrategias para incidir en lo público de acuerdo con sus relaciones entre quienes toman las decisiones en diferentes niveles del gobierno: el cabildeo y la demanda popular son parte de estas estrategias.

Ante las contradicciones que provocan las políticas económicas neoliberales en la estructura social, política, económica y cultural a escala local, es importante analizar las particularidades que asumen los procesos de cambio, adaptación y resistencia de las OSC; a saber, las nuevas formas de participación en lo público, en la organización interna, en la definición de nuevas estrategias de manifestación social. Todo esto define a las OSC como entes políticos capaces de posicionarse en lo público conforme a sus intereses.

Como antecedente para visualizar el contexto de implementación de las políticas neoliberales, es preciso decir que, en la década de 1980, los países de América Latina tuvieron que reorientar su política económica debido a una gran crisis estructural. El modelo recomendado por la Comisión Económica para América Latina (CEPAL) fue el de sustitución de importaciones; sin embargo, éste fracasó rotundamente por la ineficiente capacidad del Estado para controlar el mercado.

De acuerdo con Moreno-Brid, Pérez Caldentey, y Ruiz Nápoles (2005), desde mediados de la década de 1980, América Latina comenzó a modificar su estrategia de desarrollo: "dicha reorientación fue la respuesta a la crisis internacional de la deuda, inaugurada en 1982 y provocada por el drástico incremento del servicio de la deuda externa, combinado con la severa restricción en el acceso de América Latina al financiamiento internacional y un deterioro notable en los términos del intercambio" (p. 151).

Para el mes de noviembre de 1989, el Instituto de Economía Internacional realizó, en Washington, una reunión con ministros de economía procedentes de diversos países latinoamericanos, representantes de la Reserva Federal, del Fondo Monetario Internacional (FMI), el Banco Mundial (BM) y del gobierno de Estados Unidos, en la que John Williamson presentó el documento titulado "Lo que Washington quiere decir por reforma de la política", conocido como "Consenso de Washington", y del cual se desprenden diez recomendaciones para la política económica de América Latina. En general, con estas recomendaciones se buscaba:

1) Asegurar la disciplina fiscal, con un déficit operativo de no más del $2 \%$ del PIB

2) Establecer prioridades de gasto público, es decir, eliminar todos los subsidios

3) Incrementar el ingreso fiscal, al ampliar la base gravable y moderar las tasas marginales 
4) Liberalizar las tasas de interés

5) Establecer un régimen flexible del tipo de cambio

6) Liberalizar el comercio exterior

7) Liberalizar los flujos de inversión extranjera

8) Privatizar empresas paraestatales para conseguir más eficiencia

9) Desregular para promover la competencia

10) Garantizar los derechos de propiedad (en forma similar a la de Estados Unidos)

Estos principios facilitaron que se emprendiera en México un proceso de liberalización comercial, que a su vez abrió las puertas a la privatización de empresas estatales y, en general, propició la reducción de la intervención económica del Estado, así como un recorte importante al gasto en inversión social (Moreno-Brid, Pérez Caldentey, y Ruiz Nápoles, 2005). Tales políticas y recortes públicos originaron ajustes en el mercado interno, desempleo y grandes olas de migración, sobre todo de ciudades pequeñas hacia las grandes urbes. En este contexto, la nueva dinámica generó nuevos problemas y la emergencia de nuevos actores en la búsqueda de soluciones; es el caso de las OSC que señalaron y atendieron los problemas que enfrentaba la sociedad de Chihuahua en los años ochenta del siglo XX.

\section{LAS OSC EN CHIHUAHUA}

El contexto estatal

Ubicado al norte de México, Chihuahua colinda al norte con Texas y Nuevo México, Estados Unidos; al sur con Durango, al este con Coahuila y al oeste con Sonora y Sinaloa en la República mexicana. Chihuahua es el estado más grande del país, con una extensión territorial de 250, 673 $\mathrm{km}^{2} \mathrm{y}$, de acuerdo con datos del Instituto Nacional de Estadística, Geografía e Informática (INEGI, 2010), cuenta con 3, 406,465 habitantes (habs.). Se divide en 67 municipios, de los cuales cinco, considerados urbanos, concentran el mayor número de pobladores: Juárez alberga a 1,332,131 habs.; Chihuahua, a 819,543; Cuauhtémoc, a 154,639; Delicias, a 137,935 e Hidalgo del Parral, a 107,061 habs. (INEGI, 2010). En el resto de los municipios, considerados semi-urbanos y rurales, la población no supera los 100,000 habs.

Las ciudades de Juárez y Chihuahua - esta última, capital del Estado- se caracterizan por concentrar la mayor parte de la actividad económica y de participación política y social. Ciudad Juárez colinda con El Paso, Texas, y su ubicación geográfica la convierte en el principal polo de desarrollo, pues ahí se localiza el mayor número de establecimientos de industria maquiladora y de servicios en el Estado. 
Los datos del Prontuario Estadístico de la Secretaria de Economía del Gobierno del Estado de Chihuahua muestran que, para el tercer trimestre de 2014, la Población Económicamente Activa (PEA) era de 1.57 millones de personas, mientras que la tasa de desempleo se contaba en 4.6 millones. Los datos indican, asimismo, que en el Estado había 477 establecimientos de la Industria Maquiladora de Exportación (IMMEX), los cuales ocupaban el primer lugar nacional en personal contratado en ese sector, con 313,459 personas. En cuanto al número de personas aseguradas por el Instituto Mexicano del Seguro Social (IMSS) en el Estado, 50\% de los 753,329 asegurados habitaban en Ciudad Juárez y 30\%, en la ciudad de Chihuahua (Gobierno del Estado, 2015).

Es importante destacar que Chihuahua cuenta con 4.5\% del total nacional de la Inversión Extranjera Directa (IED), por lo que ocupa $4^{\circ}$ lugar en el país en captación de inversores y $1^{\text {er }}$ lugar nacional en exportaciones no petroleras (Gobierno del Estado, 2015). En el sector minero, para Mapa 1.

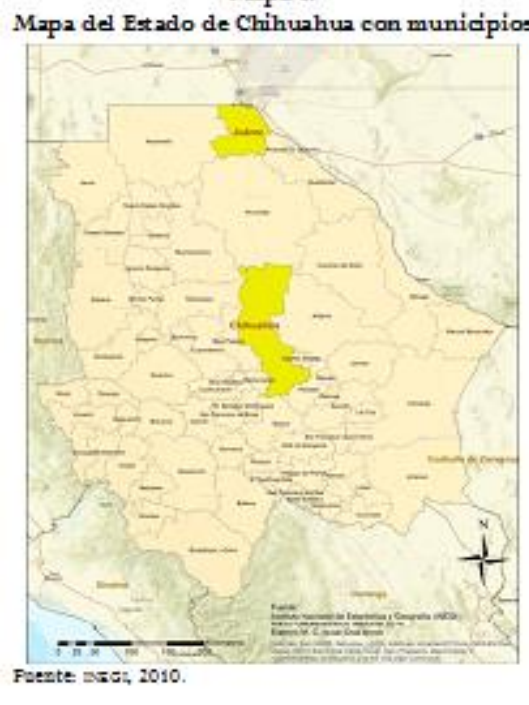
enero de 2015, el Estado se ubicaba en el $3^{\text {er }}$ lugar nacional en producción de oro y el $2^{\circ}$ en producción de plata y zinc. Además, por la misma fecha, el sector agropecuario estatal se caracterizaba por ocupar el $1^{\text {er }}$ lugar nacional en la producción de nuez, cereza, papa, manzana, algodón hueso, trigo forrajero verde, avena grano, avena forrajera y cacahuate; también, el $2^{\circ}$ lugar nacional en producción de maíz amarillo, chile verde, alfalfa verde y durazno; el $4^{\circ}$ lugar en producción de guajolote y leche bovina, y $9^{\circ}$ lugar en producción de carne bovina.

En Chihuahua, la actividad de las OSC es muy diversa; existen esfuerzos, como el de la Fundación del Empresariado Chihuahuense y de Desarrollo Social del Estado y del gobierno federal, por integrar un directorio con información categórica sobre la participación de este sector en la entidad. No obstante que los datos pueden variar de una fuente a otra, se estima que en Chihuahua hay más de 400 OSC y hay coincidencia en que la mayor actividad del sector se concentra en las ciudades de Juárez y Chihuahua, razón por la cual el análisis que presentamos se basa principalmente en esos dos municipios.

En el año 2011, el Centro para el Fortalecimiento de la Sociedad Civil (CFOSC) realizó el estudio denominado El Aporte de las Organizaciones de la Sociedad Civil (OSCS). La Dimensión del Sector Filantrópico de Ciudad Juárez". Con este proyecto, el CFOSC buscaba reunir y analizar información concreta que permitiera medir la presencia y aporte de las asociaciones civiles de Ciudad Juárez, además de conocer su situación para detectar tanto oportunidades y amenazas, como áreas de fortaleza y debilidad de las mismas (CFOSC, 2011). 
El estudio detectó que, de acuerdo con diferentes fuentes, en 2010 había diversas organizaciones sociales en el estado de Chihuahua; particularmente, en Ciudad Juárez.

Cuadro 1. Registros de Organizaciones Sociales en Chihuahua

\begin{tabular}{|c|c|c|c|}
\hline Fuente & Chihuahua & Ciudad Juárez & $\%$ del total \\
\hline Registro Público de la Propiedad del Estado de Chihuahua & 5,966 & 1,728 & $29 \%$ \\
\hline Directorio Donatarias Autorizadas (SAT) & 357 & 141 & $39.5 \%$ \\
\hline Secretaría de Desarrollo Social (CLUNI) & 375 & 101 & $26.9 \%$ \\
\hline Directorio de la Fundación del Empresariado Chihuahuense, A.C. (FECHAC) & 800 & 222 & $27.8 \%$ \\
\hline CFOSC, Ciudad. Juárez (RFC, CLUNI, Donatarias) & --- & 289 & --- \\
\hline
\end{tabular}

Fuente: CFOSC, con datos de las mencionadas instancias.

Como se puede ver en el Cuadro anterior, en el Registro Público de la Propiedad del Estado de Chihuahua consta la existencia de 5,966 asociaciones civiles, legalmente constituidas, que contemplan todo el abanico correspondiente al tercer sector: "asociaciones religiosas, asociaciones de auto beneficio, partidos y organizaciones políticas, instituciones de servicios a terceros”, de los cuales 1,728 pertenecen al municipio de Ciudad Juárez. Esto indica la importancia de diferenciar qué es una organización de la sociedad civil y qué una asociación civil propiamente, para entender que los directorios creados se apegan a diversos intereses o criterios temporales y geográficos, y llevar a cabo un registro más preciso en el presente estudio ${ }^{1}$ (CFOSC, 2011).

En 2016, con base en las fuentes arriba señaladas (el Centro para el Fortalecimiento de Organizaciones Sociales, la Fundación del Empresariado Chihuahuense y la Junta de Asistencia Social Privada del Gobierno del Estado de Chihuahua), elaboramos un directorio condensado de las OSC que operan y tienen presencia en Ciudad Juárez. Derivado de ello, y luego de eliminar las organizaciones repetidas en unas y otras fuentes, contabilizamos un total de 450 organizaciones sociales que operaban en la ciudad; unas activas y otras no; unas con registro CLUNI (236) activo (143), inactivo (93) y otras sin registro (214), que trabajan en diversos campos de acción u objetos sociales. El intento de verificar tales campos de acción se muestra en el siguiente Cuadro, con la distribución porcentual de las OSC locales, por campo, en un comparativo entre los registros de distintas fuentes de los años 2010 y 2016.

${ }^{1}$ El estudio distingue entre ambos conceptos, de la siguiente manera: 1) ¿Qué son las OsC? Agrupaciones constituidas por individuos que, a partir de lazos asociativos, realizan actividades de defensa y respeto a los derechos humanos, de apoyo o asistencia a terceros sin fines de lucro ni búsqueda de beneficios personales, sino beneficios sociales, comunitarios. Esencialmente, su origen responde al derecho de todo miembro de la sociedad a ejercer su participación ciudadana, como clave para una mayor corresponsabilidad de los gobiernos y los ciudadanos. 2) ¿Qué es una Asociación Civil? Asociación de individuos que convienen en reunirse, de manera no del todo transitoria, para conseguir un fin común, no prohibido por la ley ni de carácter preponderantemente económico. En otras palabras, es una persona jurídica privada, integrada por un conjunto de personas físicas (llamadas socios) que, con la debida autorización del Estado, se unen para realizar actividades que tienden al bien común. La característica que las distingue es que no persiguen una ganancia comercial ni económica, por ello es frecuente que se las denomine también "Entidades civiles sin fines de lucro". 
Cuadro 2.

Distribución \% de las OSC en Ciudad Juárez, por campo de acción, 2010 y 2016

\begin{tabular}{l|c|c}
\hline Campo de acción & 2010 & 2016 \\
\hline Arte, cultura y deportes & $3 \%$ & $5.77 \%$ \\
\hline Derechos humanos & $2 \%$ & $2.88 \%$ \\
\hline Desarrollo social & $15 \%$ & $15.55 \%$ \\
\hline Discapacidad & $7 \%$ & $5.33 \%$ \\
\hline Educativo & $9 \%$ & $10.22 \%$ \\
\hline Intermediarios filantrópicos & $2 \%$ & $4.88 \%$ \\
\hline Medio ambiente & $1 \%$ & $2.44 \%$ \\
\hline Rehabilitación & $15 \%$ & $7.77 \%$ \\
\hline Salud & $7 \%$ & $8.0 \%$ \\
\hline Servicios sociales & $34 \%$ & $30.88 \%$ \\
\hline Colegios de profesionistas y clubs de servicios & $6 \%$ & \\
\hline N/D & & $6.22 \%$ \\
\hline
\end{tabular}

Fuente: Fortaleza, FECHAC, INDESOL.

Con este panorama, se puede dar cuenta del arduo trabajo que se realiza en el sector social en Chihuahua.

\section{Desarrollo de las OSC en Chihuahua}

El origen de las OSC en Chihuahua puede ubicarse en dos grandes sectores; por una parte, las movilizaciones populares derivadas de los procesos electorales que iniciaron en Chihuahua en 1983 - cuando el Partido Acción Nacional (PAN) triunfó en Ciudad Juárez por primera vez en su historia-; por otra parte, un movimiento urbano-popular cuya fisonomía está ligada a las inquietudes de sectores y grupos sociales marginados del desarrollo urbano y social, que han buscado y buscan mejorar sus condiciones de vida y de trabajo.

Durante las últimas dos décadas, en Chihuahua, como en otras regiones del país, se han desarrollado corrientes sociales con perfiles que requieren de un análisis detenido y riguroso para su mejor comprensión. En general, es posible afirmar que surgieron en Chihuahua dos tipos de causas sociales que actuaron como motor para la organización de los ciudadanos: los movimientos cívico-políticos y los movimientos urbano-populares.

En el primer caso, ha predominado la naturaleza cívico-política, es decir, estructuras orientadas a combatir las prácticas "priistas" y a construir espacios democráticos. Sus principales batallas iban "contra un régimen político con monopolio del poder en un partido y a favor de una democratización en el ejercicio del poder y de la pluralidad competitiva en igualdad de circunstancias, respeto al voto, menos injerencia gubernamental en los mecanismos de renovación de poderes" (Lau y Quintana, 1991:5). En el segundo caso, el movimiento cívico-político de izquierda de los años sesenta en Chihuahua estuvo influido por la Revolución Cubana; en el Estado se manifestaron movimientos tan radicales como la Liga 23 de Septiembre. 
De acuerdo con Lau y Quintana, "fue la teoría del enfoque revolucionario lo que se impuso. Textos como ¿Revolución en la Revolución?, de Regis Debray, eran verdaderos catecismos. La Revolución Cubana propició que mucha gente comenzará a cuestionar el programa y la táctica de los partidos de izquierda de la época: el Partido Comunista Mexicano y el Partido Popular Socialista (Lau y Quintana, 1991:15).

La organización social más importante de carácter cívico-político tuvo lugar en Chihuahua durante el ciclo electoral de 1983-1988, con notoria participación de amplios sectores de la población de clase media. En ese quinquenio se presentaron las dos luchas más importantes de sectores sociales: obreros siderúrgicos y campesinos. Los trabajadores de Aceros de Chihuahua, en huelga y gestiones para adjudicarse la empresa, y las movilizaciones campesinas por mejores precios en el noroeste de la entidad; sin embargo, a pesar de ciertos esfuerzos, no se produjo una sólida articulación con la propuesta político-electoral (Lau y Quintana, 1991:7).

Cabe destacar que las formas de manifestación de la sociedad civil de ese tiempo, para cuestionar las acciones del gobierno, eran muy creativas. Además, la abierta identificación y el consiguiente rechazo de la corrupción asociada al gobierno, al Partido Revolucionario Institucional (PRI) y a la crisis económica, contribuyeron a capitalizar el malestar colectivo. En este tenor, se instrumentaron rituales prácticos, como el uso de colores en estándares y banderolas; algunas señales que comunicaban la convergencia con los propósitos políticos, como el toque del cláxones; el lanzamiento de campañas que implicaban la participación individual mediante acciones diversas, tales como no pagar el agua, voltear las placas de los automóviles, bloquear teléfonos de oficinas públicas, etc. (Lau y Quintana, 1991:8).

Además, las organizaciones sociales urbano-populares, cuyos activistas se guiaban por la ideología de la izquierda, resistían a los "efectos de la política económica, la vida sindical y el desarrollo capitalista en general, y exigían solución a demandas inmediatas” (Lau y Quintana, 1991: 6). Las distintas estructuras urbano-populares de Chihuahua, con su diversidad temática, se han guiado por la búsqueda de mejores condiciones de habitabilidad de los espacios (servicios públicos, vivienda, lotes urbanos, etc.), de trabajo (salarios y prestaciones, precios diferentes para campesinos) y sindicales (liderazgos representativos).

Una buena parte de esa problemática fue canalizada, por la vía de la gestión, modalidad asumida por el Comité de Defensa Popular (CDP), al cual se incorporó también otro organismo: el Frente Democrático Campesino (FDC), que aglutinó las demandas de los campesinos del noroeste del Estado. Tanto por su dinámica como por su naturaleza, la acción política y de gestión de ambas organizaciones se inscribió entre los complejos vínculos Estado-Sociedad civil (Lau y Quintana, 1991: 9). El CDP nació en la ciudad de Chihuahua en 1972: "sus tácticas de lucha eran la manifestación y el mitin, los cuales tenían como finalidad atraer al mayor número de personas a las filas de los inconformes con el sistema político dominante actual. Había una renuncia explícita 
y una crítica soterrada a la línea 'priista', inclinándose ante todo por las luchas de masas" (Lau y Quintana, 1991: 23).

En esencia, el CDP instaba a luchar por la democracia sindical, el aumento de los salarios, la instauración de la semana de 40 horas y la administración campesina en las instituciones de crédito relacionadas con el campo, entre otros intereses sociales. En cuanto a la problemática urbana, una de las principales propuestas consistía en expropiar terrenos destinados a la construcción de viviendas, y conseguir o gestionar el incremento de servicios públicos tanto para las colonias que se estaban creando, como para las ya edificadas que carecían de ellos.

Años más tarde, en la década de los noventa, cuando, por una parte, los fraudes electorales dejaron de ser prioritarios y, por otra, la alternancia política en el Estado y en algunos municipios había dejado claro que el cambio de partidos en el gobierno no era la solución, ni inspiraba ya una movilización social, la vocación de las OSC se reorientó a subsanar problemas, en muchos casos, no atendidos por el Estado.

En el nuevo milenio, el panorama no es muy distinto. La problemática de las principales ciudades del estado de Chihuahua continúa siendo propicia para la participación de las OSC, en tanto actores que buscan soluciones para atenuarla. Es de suma importancia destacar que durante la última década, y más precisamente a partir de 2007, el Estado ha atravesado por una serie de crisis políticas, económicas, sociales y de inseguridad, que también han trasminado la dinámica de las OSC.

\section{Desarrollo de las OSC en Ciudad Juárez}

En Ciudad Juárez, el origen de las OSC es muy diverso, y va desde las comunidades eclesiales de base, hasta los grupos de colonos hastiados de las prácticas clientelares del PRI, que decidieron organizarse para gestionar servicios básicos, terrenos, pavimentación, drenaje, etc. En este proceso participó, inclusive, el movimiento contra el fraude electoral de 1986.

La sociedad civil se caracteriza por su heterogeneidad en cuanto a las opiniones que emite al espacio público y sus formas de acción colectiva; por cuanto toca a éstas últimas, es posible hablar de organizaciones sociales vinculadas a ideologías de izquierda y derecha. Pese a aquéllas. ambas ideologías han pugnado por la consolidación de mecanismos institucionales que les permitan incidir en la agenda pública, lo que ha derivado en la posibilidad de crear marcos normativos para

incidir públicamente. Entre estos mecanismos se hallan los Reglamentos de Órganos de Participación Ciudadana y el Reglamento del Consejo Municipal de Desarrollo Social y Participación Ciudadana.

En Ciudad Juárez, los fenómenos de violencia contra las mujeres (discriminación, machismo, asesinato), la inseguridad pública, la carencia de servicios básicos, los problemas de contaminación, la escasez de espacios culturales, la falta de credibilidad en el gobierno por parte 
de la ciudadanía, el narcotráfico y, en general, la delincuencia organizada, entre otros factores, se han agudizado en los últimos treinta años, obligando a la sociedad civil a formar grupos interesados y dispuestos a demandar y gestionar, ante instancias de gobierno, la solución de esas problemáticas.

La emergencia de grupos organizados en Ciudad Juárez, desde finales de los años setenta, se encuentra asociada a tres momentos estratégicos; primero, la necesidad de impulsar medidas protectoras para las y los trabajadores de las maquiladoras; segundo, la pugna por reformas legislativas para la persecución de delitos relacionados con la violencia en contra de las mujeres; tercero, la gestión, ante el municipio, de posibles soluciones a los problemas urgentes de equipamiento público y servicios urbanos.

El primero y segundo momentos forman parte del movimiento de lucha por los derechos de las y los trabajadores, y la necesidad de una ley que protegiera los derechos de las mujeres en Ciudad Juárez, lo que "tiene su origen en un activismo militante de sectores académicos y sindicalistas que luchan por la defensa de los derechos de las trabajadoras de la maquila. Y surge prácticamente desde fines de los setentas y principios de los ochentas y se mantiene hasta la actualidad" (Ravelo, 2004:22).

Una de las primeras iniciativas para crear una instancia de defensa de los derechos laborales en Ciudad Juárez fue el Centro de Orientación de la Mujer Obrera (COMO), creado en 1973 y dirigido por Guillermina Valdés Villalba; otra es el "Comité Feminista 8 de Marzo", fundado por Esther Chávez Cano, quien en 1998 instauró el Centro de Crisis "Casa Amiga"; una más es "Nuestras Hijas de Regreso a Casa” (NHRC), instituida en el año 2002 por Marisela Ortiz y Rosario Acosta. Existen otros dos grupos de esta naturaleza: el Centro de Estudios y Taller Laboral A.C. (CETLAC), formado hacia fines de 1996, y el Centro de Investigación y Solidaridad Obrera, A.C. (CISO), de más reciente creación, en 2000 (Ravelo, 2004:22).

Otro grupo de organizaciones creadas en el pasado para promover los derechos de las mujeres incluyó a "Mujeres por Juárez" (1990), el Centro para el Desarrollo Integral de la Mujer y el Comité de Lucha contra la Violencia (1993), así como el Centro de Mujeres Tonantzin (1997), Voces sin Eco y Red de Mujeres, fundados en 1998.

El tercer momento estratégico tiene su origen en los grupos constituidos desde finales de los años ochenta; todos con importantes trayectorias, experiencia de trabajo y militancia política, alejados de las estrategias tradicionales utilizadas por el PRI. Los temas a que se orientaron fueron la promoción de los derechos humanos, la orientación sindical y la educación laboral, así como el desarrollo comunitario, la salud integral, la lucha contra la violencia y la atención a mujeres viviendo con VIH/Sida y sus familias.

Entre los grupos que nacieron con el objeto de contribuir a solucionar esas problemáticas, destaca la Organización Popular Independiente (OPI), que surge de las comunidades eclesiales de 
base en 1987, como un movimiento social de protesta contra el aumento del precio de la tortilla; posteriormente, se convirtió en una organización alternativa a las estrategias de los "priístas". La OPI ha diversificado sus objetivos y actualmente está centrada en la gestión de educación y capital social en las colonias de mayor marginación al poniente de Ciudad Juárez. Otras organizaciones son el Programa Compañeros A.C., constituido en 1987, y Salud y Desarrollo Comunitario (SADEC), que data de 1993.

De reciente creación, identificamos Casa Promoción Juvenil (1996), que apoya a jóvenes en condiciones de riesgo social; la Federación Mexicana de Asociaciones Privadas de Salud (FEMAP), dedicada a la promoción de la salud, y la Red del Consejo Ciudadano para el Desarrollo de Ciudad Juárez, creada en el año 2004 y conformada por 40 organizaciones que, divididas en mesas de trabajo, intervienen en diversas áreas de la ciudad. Entre las principales mesas que existen actualmente, se cuentan: "Infancia”, “Jóvenes", "Mujeres”, "Migrantes”, "Indígenas" y "Adultos Mayores".

\section{ESTRATEGIAS DEL ESTADO Y AGOTAMIENTO DEL MODELO DE ORGANIZACIÓN}

Las posturas académicas de quienes han estudiado el fenómeno de las OSC en Ciudad Juárez hacen referencia a varios aspectos; por ejemplo, Ravelo (2004) argumenta que las Organizaciones No Gubernamentales (ONG) llegaron a su fin por contener un modelo asistencialista; Pérez (2005) argumenta que fue el propio gobierno quien las desprestigió; Rojas (2005) presenta una sociedad civil reducida al espacio de lo privado por las estructuras machistas prevalecientes entre los gobernantes, y Bustillos (2004) refiere organizaciones pragmáticas que socavan el potencial democrático de las comunidades locales, ya que únicamente se representan los valores e intereses de unos cuantos.

De acuerdo con la teoría sobre la sociedad civil, en 1996 la frontera ofrecía "alternativas para que la gente pudiera comunicarse, organizarse, promover o defender sus intereses en la esfera pública" (Denman y Caudillo, 1996:19). No obstante, si analizamos la propuesta teórica de algunas intelectuales sobre ese hecho social, los datos son completamente diferentes. Investigadoras locales y nacionales afirman que la figura de la sociedad civil está desapareciendo; en particular, la de las ONG, actualmente denominadas OSC, debido a que algunas de ellas han adoptado un modelo asistencial y reproductivo, además de que pocas o ninguna se pueden considerar, francamente, líderes sociales contra-hegemónicos.

Sin embargo, aparte del modelo asistencial de las ONG, lo que está en decadencia es su paradigma de adscripción; esto es, no cuentan con una teoría política que cambie el modelo neoliberal. En los campos de trabajo en género y medio ambiente, se observa esta tendencia; por ejemplo, Ravelo (2004) identifica la problemática de algunas OsC de Ciudad Juárez, a las que 
considera "desgastadas". No sabemos porque realmente no han logrado desligarse del gobierno, dice:

incluso no sabemos si se tenga conciencia de los alcances de la cooptación gubernamental que han experimentado algunas organizaciones, representantes y madres de mujeres asesinadas; si la "carrera" política que han hecho las dirigencias ha sido la correcta, pues sus organizaciones no han dejado de tener un carácter asistencialista, se han profesionalizado de manera desigual, mantienen estructuras jerárquicas y, en algunos casos, parece que se aprovechan de la situación de violencia para obtener beneficios personales (p. 21).

Pérez García considera, por su parte, que al llegar al gobierno Patricio Martínez (1998-2004) se olvidó de sus promesas de campaña, en el sentido de terminar con los asesinatos y encontrar a los culpables; adoptó una actitud retadora y creó una lucha de poder; se alejó de las ONG, lo cual empezó a desordenar el movimiento; su línea hacia los grupos generó un ambiente de tensión y creó problemas internos, lo que terminó por exhibir ante la comunidad sus principales vulnerabilidades (Pérez, 2005:164).

Rojas Blanco (2005) considera que, debido a la retórica del menosprecio a las mujeres en Ciudad Juárez, fue difícil entender el papel de la sociedad civil en lo que refiere al feminicidio, y plantea que:

el reconocimiento de las mujeres como agentes activos depende, en primera instancia, de la posibilidad y habilidad, por parte de las agentes involucradas, de articular, desarticular y rearticular el poder simbólico de la voz y el silencio utilizado como estrategias políticas. por otro, digo que durante los procesos de (re)negociación política, la voz y el silencio rebasan las posibilidades lingüísticas o metafóricas para convertirse en prácticas significantes" (p. 16).

De acuerdo con Rojas, el feminicidio sorprendió a la sociedad civil, dejándola sin respuestas, "sin agenda feminista; nos abofetearon los históricos silencios relativos a los derechos de las mujeres en esta frontera. Nos paralizó el uso del lenguaje” (p. 16). El fenómeno de las ONG no pudo responder a la magnitud del fenómeno del feminicidio por las imbricaciones machistas de los gobernantes.

Desde la perspectiva de Bustillos (2004), en el mediano y largo plazo, las formas de operar de las ONG tienen el efecto de "socavar en mucho el potencial democrático de las comunidades locales, ya que únicamente se representan los valores e intereses de unos cuantos. Más allá de reflejar diferencias sociales, la promoción desigual por parte de las ONG (su conformación misma) encubre divisiones, promueve el faccionalismo y exacerba la marginación de grandes grupos sociales (p. 76). 
Es necesario reconocer que no todas las OSC sufren los mismos cambios en sus estructuras operativas debido al Neoliberalismo; esto depende de la capacidad de posicionamiento y negociación con que hayan contribuido en el campo de lo político y lo económico, tanto a escala regional como en otra medida. Otro factor que interviene en el proceso de resistencia y adaptación al Neoliberalismo deriva del contacto que guardan las OSC con la comunidad; depende de la relación que establezcan en el tiempo y el espacio con sus comunidades inmediatas, así como de la red relacional con otras OSC.

Desde una óptica multicausal, el agotamiento del modelo de las ONG deviene, en principio, de tres fenómenos: primero, las OSC empezaron a recibir recursos financieros en la década de los ochentas, lo cual provocó su distanciamiento con la comunidad (Adopción de un modelo asistencial); segundo, las demandas sociales iniciaron un proceso de institucionalización entre el Estado y el ciudadano (Institucionalización de demandas) y, tercero, la ideología en la cual se apoyaron las OSC fue sustituida por el paradigma neoliberal. En estas circunstancias, surgió un fenómeno de agotamiento de los paradigmas. Los tres fenómenos convergen en la institucionalización de las OSC, de la siguiente manera:

a) Con la Adopción de un modelo asistencial por parte de las ONG, actualmente existen organizaciones que tuvieron un origen reivindicativo y/o de movimiento social, es decir, que demandaba servicios básicos, viviendas, terrenos, derechos humanos, derechos de la mujer etc.; sin embargo, con el tiempo sus propuestas se institucionalizaron al incorporar capacidades para concursar y adquirir recursos financieros de fundaciones nacionales e internacionales y de los tres niveles del gobierno, con lo cual la promoción del desarrollo social pasó a un segundo término.

b) La Institucionalización de demandas, proceso mediante el cual las convocatorias para concursar por recursos se convierten en el vínculo entre el Estado-mercado y las OSC, significa que las necesidades reales y sentidas de las comunidades dejan de ser consensuadas al interior de las mismas, trasladando a las instituciones del Estado y a las fundaciones la responsabilidad de proponer los temas en las convocatorias.

En 2004 se publicó, en el Diario Oficial de la Federación, la Ley de Fomento a las Actividades Realizadas por las Organizaciones de la Sociedad Civil (LFAROSC). En esta legislación, se decretó la creación de un Consejo Consultivo encargado de diseñar los temas de las convocatorias dirigidas a las OSC del país, vía el Instituto Nacional de Desarrollo Social (INDESOL). Esta normativa se creó para reconocer y favorecer la presencia pública de las OSC; sin embargo, ha sido utilizada por el INDESOL como un mecanismo de control de áreas de trabajo, que, en general, funciona más bien como medio para institucionalizar las demandas sociales.

La LFAROSC ha permitido reglamentar la acción colectiva de las OSC, pues, mediante la creación del padrón de control, ha transformado los procedimientos ciudadanos de manifestación 
y demanda, acelerando el proceso de institucionalización. Para ello, existen indicadores diseñados por el Centro Mexicano para la Filantropía (CEMEFI), que miden los niveles de institucionalidad y transparencia. Cabe mencionar que si las OSC no cumplen con alguna de las citadas regulaciones, se ven impedidas para obtener financiamiento de instituciones públicas o privadas.

El CEMEFI (2007) cuenta con diez niveles para medir la institucionalidad y la transparencia de las OsC:

1. Contar con Acta Constitutiva registrada ante notario

2. Contar con autorización de la Secretaría de Hacienda y Crédito Público (SHCP) como donataria

3. Misión, visión y objetivos establecidos

4. Domicilio y teléfono verificables

5. Elaborar informe anual de actividades y resultados

6. Contar con un Consejo Directivo diferente al equipo profesional

7. Contar con personal profesional contratado en nómina

8. Contar con voluntarios trabajando en los programas

9. Tener más de tres años de operación

10. Financiamiento de más de tres fuentes distintas de ingresos

De estos puntos, la institucionalización básica incluye los primeros cinco indicadores, y los de mayor importancia para las OSC son la constitución legal y fiscal, la claridad en la enunciación de misión, visión y objetivos, así como el ejercicio de una administración transparente.

Un nivel medio de institucionalización corresponde a las organizaciones que alcanzan entre seis y ocho indicadores. Se considera la buena administración (personal operativo y voluntario profesional trabajando en programas bien definidos) como el indicador para alcanzar el éxito en la gestión de las OSC.

En cuanto al nivel óptimo de institucionalización, éste corresponde a aquellas instancias que alcanzan nueve o más de los indicadores: "este nivel es el resultante de contar con una institución con un "alto impacto" en la comunidad" (CEMEFI, 2004:5).

c) El Agotamiento de los paradigmas se refiere al fenómeno en que la competencia entre organizaciones, por los recursos financieros, altera la estructura en el campo político donde coinciden las OSC y, por ende, deriva en la calidad de la democracia al intervenir en los proceso de construcción interna.

Los paradigmas Teoría de la Liberación, Liberal y Marxista han sido sustituidos por el modelo Neoliberal que actualmente orienta la acción colectiva de algunas OSC. La dinámica de estas instancias gira en el orden de una dicotomía entre la adaptación y la resistencia, donde la adaptación resulta el camino de menor esfuerzo; la forma ideal de lograr esto es registrarse ante el 
INDESOL y la SHCP, pero sus implicaciones son de carácter institucional, ya que este tipo de medidas conduce al distanciamiento entre la base comunitaria y los líderes sociales, por las restricciones que impone a la capacidad para resolver problemáticas sociales, además de su enfoque en las convocatorias para tener acceso a los recursos.

Lo anterior corrobora la idea de James Petras (2000), en el sentido de que la ideología de las OSC, en cuanto a sus actividades privadas y voluntarias, destruye el sentido de lo "público". Contra esta noción de responsabilidad pública, las OSC fomentan la idea neoliberal de la "responsabilidad privada" de los problemas sociales, así como la importancia de los recursos financieros para resolverlos. De tal suerte, el Estado impone una doble carga a los pobres: pagar impuestos para financiar a un Estado neoliberal que sirve principalmente al capital, y el auto explotarse de manera privada para satisfacer sus necesidades inmediatas (p. 1).

\section{UNA OSC CON INCIDENCIA}

Además del esfuerzo de profesionalización, las OsC de Chihuahua han optado por un proceso de evolución por el rumbo de la incidencia social. El concepto de incidencia identifica en sí una serie de elementos; a saber: capacidades de la organización, políticas públicas, agenda pública, agenda de gobierno y espacio público. Entendido de esta manera, a continuación exponemos un ejemplo de incidencia social en el gobierno por parte del Plan Estratégico de Juárez (PEJ).

PEJ es una organización civil que surge con una doble tarea; la primera, construir la organización y, la segunda, iniciar el proyecto de Plan Estratégico de Juárez, impulsando acciones participativas en coordinación con el gobierno, con la pretensión de involucrar a la ciudadanía en su elaboración (Parés, 2009). Lucinda Vargas (2015), primera directora de PEJ, lo describe como un proyecto integral donde la economía y el desarrollo de la ciudad se pudieran imaginar, a largo plazo, con la participación de toda la ciudad.

Desde su inicio, la organización contó con un Grupo Visión, integrado por un Comité Director y un grupo de socios impulsores, representantes del gobierno estatal, local, empresarios y académicos, así como con un grupo de socios numerarios, es decir, personas o instituciones que participaron con una aportación económica anual e interesadas en sumarse a la Asociación Civil (PEJ, 2003).

Quienes estuvieron al frente del primer grupo -el Grupo Visión- tenían características no exactamente relacionadas con estar al frente de alguna institución: les caracterizaba su interés por la ciudad. En el grupo se incluía al entonces presidente municipal de Ciudad Juárez (Gustavo Elizondo) y al gobernador del Estado de Chihuahua (Patricio Martínez), ambos de distintos partidos políticos. Es importante destacar la forma en que se estructuró la organización de PEJ, 
pues su composición le garantizó tres elementos, por lo menos: autofinanciamiento, liderazgo y continuidad.

Por lo que se refiere a la autofinanciación, el grupo de socios impulsores ofreció un respaldo económico que avalaba a la organización, en cuanto al grado de independencia ideológica y política, por lo menos con respecto al partido en el poder. La solvencia económica influyó, a su vez, en los otros elementos de la organización; en cuanto al liderazgo, la organización aglutinaba a varios de los líderes económicos, políticos y morales de la ciudad. El esfuerzo de reunir a los diversos grupos de la estructura organizativa, incluía la presencia de instituciones académicas de reconocido prestigio, de empresarios filantrópicos y de varias personalidades de diversas corrientes ideológicas, líderes en sus ámbitos, e inclusive la mayoría de ellos ubicados en espacios estratégicos para la toma de decisiones en diversos sectores de la ciudad.

Con el respaldo económico y la visión de líderes sociales, políticos y económicos, se viabilizó el elemento de continuidad del Plan. El diseño, integración y puesta en marcha fue de alguna manera supervisado y avalado por los diversos actores dentro del PEJ. Inclusive, la etapa de adaptación a los cambios, cuando el PEJ cambió el enfoque de un plan integral por un enfoque dirigido a la gobernabilidad, la anuencia de los diversos grupos fue factor clave para la continuidad y el replanteamiento del quehacer de la organización.

El diseño original del PEJ se desarrolló con base en herramientas para la participación ciudadana. La innovación, al respecto, consistió en la operacionalización masiva de herramientas de investigación cualitativa.

1. Entrevistas con actores estratégicos

2. Foros de diagnóstico: Paneles de expertos

3. Foros de diagnóstico: Mesas de trabajo en la comunidad

4. Grupo de enfoque

5. Taller: Ciudad del conocimiento

6. Encuesta a hogares

Con la participación de diversos actores, en cada una de las experiencias se logró integrar la visión de Ciudad Juárez hacia el año 2015, que plasmaba, en tres ejes transversales y cuatro ejes estratégicos, los elementos para lograr una ciudad con calidad de vida. También se logró fomentar la participación ciudadana y la conciencia del poder individual y de unión. Estos "fueron los primeros esfuerzos, no se tenía ninguna experiencia ni fórmula" (Vargas L., Memorias del Plan Estratégico de Juárez, 2015) para trabajar y tratar de incidir. Se pensaba en productos a largo plazo, que se convirtieron en una de las principales causas de descalificación al PEJ: la falta de acciones específicas a corto plazo. 
La demora del proceso de planeación del PEJ se convirtió en un ejercicio de reflexión continua entre toda la estructura de la organización. Actualmente, el PEJ (2014) basa su actividad en tres proyectos:

1. Así estamos Juárez: sistema de indicadores cuyo objetivo es medir la ciudad a partir de información que permite dar cuenta de cómo estamos y hacia dónde vamos

2. Red de Vecinos: grupo de representantes de vecinos de diferentes sectores de Ciudad Juárez, interesados en participar en cuestiones que afectan su contexto inmediato. Sus objetivos son:

- fomentar y fortalecer la participación vecinal

- proponer soluciones para nuestra ciudad

- incidir en la toma de decisiones en la ciudad

Actualmente la red tiene presencia en las colonias Calzada del Río, Pedro Rosales de León, Pradera Dorada, Valle de Bravo y Valle del Sol.

3. Regidor 19: integra a ciudadanos voluntarios para seguir y evaluar el trabajo del Cabildo de Ciudad Juárez, con el objeto de que la vigilancia se refleje en ciudadanía mejor informada y gobiernos con mejores resultados (PEJ, 2014).

Conjuntamente o a la par de tales proyectos, se trabaja en nuevas estrategias para la incidencia, es decir, para influir en las acciones que repercuten directamente en las políticas implementadas por el gobierno (Tapia, Campillo, Cruickshank, \& Morales, 2011). Con el aprendizaje derivado de los contratiempos, desavenencias y decepciones en el trabajo OSC-gobierno, se ha logrado una actuación con conciencia para influir en la disposición de recursos y en la toma de decisiones (World Bank, 1996).

La toma de conciencia se orienta hacia la búsqueda de estrategias pertinentes y a explorar nuevas maneras de cumplir con los objetivos; de tal suerte, la organización explora las estrategias legales para el interés público, en particular, el litigio estratégico, también conocido como "litigio paradigmático" o "litigio de interés público" o "de causas justas", una "herramienta de pericia de los abogados, con la cual contribuyen al Derecho de Interés Público" (OACNUDH, 2007). Este tipo de litigios ya ha sido utilizados en otras causas en México; en Chihuahua lo utiliza una OSC por primera ocasión.

La dinámica de esta estrategia está sujeta a la dinámica jurídica, es decir, a tiempos definidos conforme a un proceso legal, y los asuntos que a la fecha se han abordado mediante ella son apertura del Cabildo, revocación de decisiones que afectan el interés público, irregularidades en el cumplimiento de reglamentos municipales, y falta de transparencia en la información. 
Actualmente, esta OSC aprovecha el alcance de las Tecnologías de la Información y la Comunicación (TIC) para mantener contacto con la ciudadanía y facilitar la tarea de informar, difundir e influir en la opinión pública, para luego incidir en la toma de decisiones.

\section{REFLEXIONES FINALES}

Hay campos de la política pública municipal que carecen de mecanismos e instancias de participación; los más evidentes son la economía y la seguridad pública. Es importante problematizar la necesidad de crear instancias de participación. La intención no es sustituir al gobierno municipal por instituciones ciudadanizadas ni complicar la capacidad o atribuciones gubernamentales, sino hacer más eficiente, eficaz, sustentable y efectiva la política económica, social, cultural y de seguridad en el marco de gobernanza, es decir, donde se involucran los ciudadanos; sin su participación y la de las OSC, la democracia siempre será cuestionada.

Es necesario crear instancias eficientes y efectivas de y para la participación ciudadana en todos los ámbitos; que éstas tengan realmente funciones, atribuciones y capacidad para contribuir a la definición de las políticas públicas; de lo contrario, los mecanismos de intervención plebiscito, referéndum, iniciativa popular y revocación de mandato- son únicamente simulación, no ayudan a la gobernabilidad ni a la generación de confianza en las instituciones de gobierno.

Las áreas en que deben participar las OSC son vigilancia y cumplimiento de los derechos humanos; buen gobierno y acuerdos y tratados firmados con la administración en turno. Para las instancias civiles, esto significa vigilar la continuidad y el cumplimiento de los proyectos y programas que se implementen en los tres niveles de gobierno, desde la perspectiva de un buen sistema político y social.

Temas como discapacidad, migrantes y/o creación de escuelas representan un desgaste político para las OSC, puesto que deben ocuparse de fondear recursos en diferentes instituciones nacionales o internacionales. Asimismo, estos asuntos distraen a la sociedad civil, pues implican que sean los ciudadanos quienes vigilen las acciones de los gobiernos, que incluso responden a demandas aisladas, no consensuadas. Además, los recursos económicos y humanos de las OSC resultan insuficientes para atender áreas en las que el Estado y sus instituciones han mostrado escasa eficiencia; por ejemplo, en atención a la invalidez física y mental, a migrantes, a personas viviendo con $\mathrm{VIH}$, etc. Al respecto, lo que se hace más evidente es la institucionalización de las organizaciones de la sociedad civil, así como la implementación del modelo asistencial.

A la fecha, las OSC no han podido construir una relación de iguales entre el Estado y la sociedad civil; el modelo asistencial deja muy poco para transformar esta relación. Es necesario pensar en la construcción de un paradigma político que oriente y vincule a diferentes instancias civiles para crear una estrategia conjunta de abordaje de las funciones de que debe 
responsabilizarse. Es imperativo pensar en un una sociedad civil que haga contrapeso al Estado; en otras palabras, es pertinente consolidar una población crítica y vigilante de los gobiernos en turno.

En estos días, la sociedad civil carece de marco teórico y metodológico para exigir la solución de sus problemas; su acción se fundamenta en el sentido común y en lo simbólico. Además, su idea de emancipación ha quedado excluida. Vivimos un momento en que los problemas colectivos se resuelven desde la perspectiva de la diferencia; por lo tanto, creemos necesario construir un observatorio ciudadano, que vigile las acciones y las políticas públicas del gobierno a nivel municipal, estatal y federal.

En el contexto actual, los esfuerzos de incidencia de las OSC resultan disimiles y varían de una organización a otra. No obstante, hay ejemplos exitosos que pueden convertirse en modelos para otras organizaciones; tal es el caso del PEJ.

\section{REFERENCIAS}

ANDRÉ, P. W., with the collaboration of P. Martin and Lanmafankpotin, G. (2012). Citizen Participation. In L. CÔTÉ \& SAvard, J.-F. (Eds.), Encyclopedic Dictionary of Public Administration. Retrieved from: https://goo.gl/YG3WB7www

ARnsteIn, S. R. (1969). A Ladder Of Citizen Participation. Journal of the American Institute of Planners, 35(4), pp. 216-224. doi:10.1080/01944366908977225

AyUnTAmiento DE JuÁreZ (1998, octubre 10). Reglamento Interior del Honorable Ayuntamiento del Municipio de Juárez. México.

Bustillos DuRÁn, S. (2004). Territorio, instituciones y actores sociales en la gestión del agua en Cd. Juárez, Chih. Tesis de doctorado. Juárez: Universidad Autonoma de México (UNAM).

Centro para el Fortalecimiento de la Sociedad Civil (CFOS) (2011). El Aporte de las Organizaciones de la Sociedad Civil (OSCS). La Dimensión del Sector Filantrópico de Ciudad Juárez. México: CFOS, A.C. Retrieved marzo 16, 2016, from: $\underline{\text { https: / / goo.gl/jj8VCS }}$

Comisión de Fomento de la Actividades de OSC (2016). Buscador de OSC. México. Retrieved marzo 16, 2016, from: https: / /goo.gl/BdpujN

Denman, C. (1996). Las organizaciones no gubernamentales en la frontera México-Estados Unidos: entre el fortalecimiento comunitario y el desarrollo institucional. BorderLines 20, $4(1)$.

Directorio de las Organizaciones de la Sociedad Civil de Chihuahua (2016). Retrieved marzo 16, 2016, from https: / / goo.gl/JWvYuR

Francés García, F. J., García García, J. T., \& SAntacreu Fernández, Ó. A. (2007). Modelos participativos e innovación democrática local en los municipios valencianos. Madrid. 
FUERTE, R. (2015, 19 mayo). Acciones de coyuntura de PEJ. (C. Sarabia, Interviewer).

Gobierno del Estado de Chihuahua (2015, enero). Prontuario Estadístico. Retrieved marzo 17, 2016, from https: / / goo.gl/Trggxw

Grau, M., Moreno, E., SAnZ, J., \& IÑIgUeZ-RuedA, L. (2009). Las distintas concepciones de democracia en el mundo local y sus efectos en la participación ciudadana: la definicion de escenarios posibles. In Parés, M., Participación y calidad democrática (pp. 55-76). Barcelona: Ariel.

INEGI (2010). Censo de Población y Vivienda 2010. México. Retrieved from https: / / goo.gl/KrVJRW

Lau, R., \& Quintana Silveyra, V. M. (1991). Movimientos Populares en Chihuahua, Estudios Regionales 3. Ciudad Juarez: Universidad Autónoma de Ciudad Juárez.

Moreno-Brid, J. C., Pérez Caldentey, E., \& Ruiz NÁpoles, P. (2005, diciembre). El Consenso de Washington: aciertos, yerros y omisiones. Perfiles Latinoamericanos, pp.149168.

NABATCHI, T. (2012). A manager's guide to evaluatin citizen participation. Nueva York: Syracuse University-IBM Center for The Business of Government .

Oficina del Alto Comisionado de las Naciones Unidas para los Derechos Humanos (2007). El litigio estratégico en México: la apliación de los derechos humanos a nivel práctico. México.

OCDE (2004). Fundaciones Filantrópicas y Cooperación al Desarrollo. París.

Observatorio Internacinal de la Democracia Participativa (2015). Retrieved marzo 20, 2015, from https: / goo.gl/sXzYwW

PARÉs, M. (2009). Participación y calidad democrática. Barcelona: Ariel, Generalitat de CatalunyaDepartament d'Interior, Relacions Institucionals i Participació.

Plan Estrategico de Juarez A.C. (2015, junio 1). Regidor 19. Retrieved from www.planjuarez.org/

. (2014). Plan Estratégico de Juárez. Retrieved febrero 05, 2016, from http: / / planjuarez.org/ . (2012, agosto). Tercer Informe FECHAC. Ley de participación ciudadana de Chihuahua. Juárez, México: PEJ.

- (2012, febrero-mayo). Segundo Informe FECHAC. Ley de Participación ciudadana en Chihuhaua. Juárez, Chihuahua, Chihuahua: Plan Estratégico de Juárez.

. (2012, enero). Primer informe FECHAC. Ley de participacion ciudadana de Chihuahua. Juárez, México: PEJ.

. (2007). MANIFIESTO por la gobernabilidad de Juárez. Juárez, México: PEJ.

. (2004, septiembre). La propuesta. Juárez, Chihuahua. México: PEJ.

. (2003). Quién es Juárez? elementos de reflexión. PEJ. 
. (2003). EL Juárez que queremos. Juárez, Mexico: Sociedad de Consultoría de Gestión de la Información (SOCINTEC), PEJ.

PÉreZ GarcíA, M. E. (2005). Las Organizaciones No Gubernamentales en Ciudad Juárez y su lucha contra la violencia de género. Nóesis, 15(28), pp.147-167.

PETRAS, J. (2000, marzo 8). Las dos caras de las ONGs. La Jornada.

Gobierno Municipal de Ciudad Juárez (1998). Plan Municipal de Desarrollo 1998-2001. Juárez, México.

Ravelo Blancas, P. (2004, mayo-junio). Entre las protestas callejeras y las acciones internacionales. Diez años de activismo por la justicia social en Ciudad Juárez. El Cotidiano, 19(125), pp. 21-32.

Rousseau, J. J. (1999). El contrato social. México: El aleph.

SOCINTEC \& PEJ (2003, diciembre). El Juárez que queremos. Juárez, México.

SosA, L. D. (2014, mayo 26). Pagan juarenses $\$ 600$ mil en 7 meses en seguridad para Teto. Diario. Retrieved marzo 03, 2015, from https: / / goo.gl/RzjgdY

Tapia, M., Campillo, B., Cruickshank, S., \& Morales, G. (2011). Manual de Incidencia en políticas públicas. México: Alternativas y Capacidades A.C.

VArGaS, L. (2015, abril 9). Memorias de Plan Estratégico de Juárez. México. . (2015, abril 1). Memorias del Plan Estratégico de Juárez. (C. Sarabia, \& A. Paniagua, Interviewers).

. (2014, noviembre 7). Regresa ‘Teto' escoltas asignadas por Municipio. NORTE. Retrieved febrero 09, 2015, from https: / /goo.gl/qt536W

World Bank (1996). The World Banck Participation sourrcebook. World Bank. 